\section{Biomedical Image Denoising Based on Hybrid Optimization Algorithm and Sequential Filters}

\author{
Yousefi Moteghaed N. ${ }^{1 \oplus}$, Tabatabaeefar M..$^{2}$, Mostaar \\ A. ${ }^{1,3 *} \bullet$
}

\begin{abstract}
Background: Nowadays, image de-noising plays a very important role in medical analysis applications and pre-processing step. Many filters were designed for image processing, assuming a specific noise distribution, so the images which are acquired by different medical imaging modalities must be out of the noise.

Objectives: This study has focused on the sequence filters which are selected by a hybrid genetic algorithm and particle swarm optimization.

Material and Methods: In this analytical study, we have applied the composite of different types of noise such as salt and pepper noise, speckle noise and Gaussian noise to images to make them noisy. The Median, Max and Min filters, Gaussian filter, Average filter, Unsharp filter, Wiener filter, Log filter and Sigma filter, are the nine filters that were used in this study for the denoising of medical images as digital imaging and communications in medicine (DICOM) format.

Results: The model has been implemented on medical noisy images and the performances have been determined by the statistical analyses such as peak signal to noise ratio (PSNR), Root Mean Square error (RMSE) and Structural similarity (SSIM) index. The PSNR values were obtained between 59 to 63 and 63 to 65 for MRI and CT images. Also, the RMSE values were obtained between 36 to 47 and 12 to 20 for MRI and CT images.

Conclusion: The proposed denoising algorithm showed the significantly increment of visual quality of the images and the statistical assessment.
\end{abstract}

Citation: Yousefi Moteghaed N, Tabatabaeefar M, Mostaar A. Biomedical Image Denoising Based on Hybrid Optimization Algorithm an Sequential Filters. J Biomed Phys Eng. 2020;10(1):83-92 doi: 10.31661/jbpe.v0i0.1016.

\section{Keywords}

Medical Imaging; Denosing; Image Processing; Algorithms; Optimization

\section{Introduction}

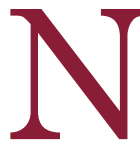

owadays medical imaging modalities such as magnetic resonance imaging (MRI) and computed tomography (CT) provide the images as precise diagnostic tools. MRI and CT scans are the most widely used methodologies to visualize human anatomy. Medical images often need pre-processing before being represented as diagnostic tools. Image denoising is one of the usual procedures in digital image processing, and it has an important role as a preprocessing step in various applications. The development of denoising algorithms is a very challenging issue because the diagnostic information must be pre-
${ }^{1} \mathrm{PhD}$, Department of Medical Physics and Biomedical Engineering, School of Medicine, Shahid Beheshti University of Medical Sciences,

Tehran, Iran

${ }^{2} \mathrm{MD}$, Department of Ra-

diation Oncology, School of Medicine, Shahid

Beheshti University of Medical Sciences, Tehran, Iran

${ }^{3} \mathrm{PhD}$, Radiation Biology

Research Center, Iran

University of Medical Sciences, Tehran, Iran

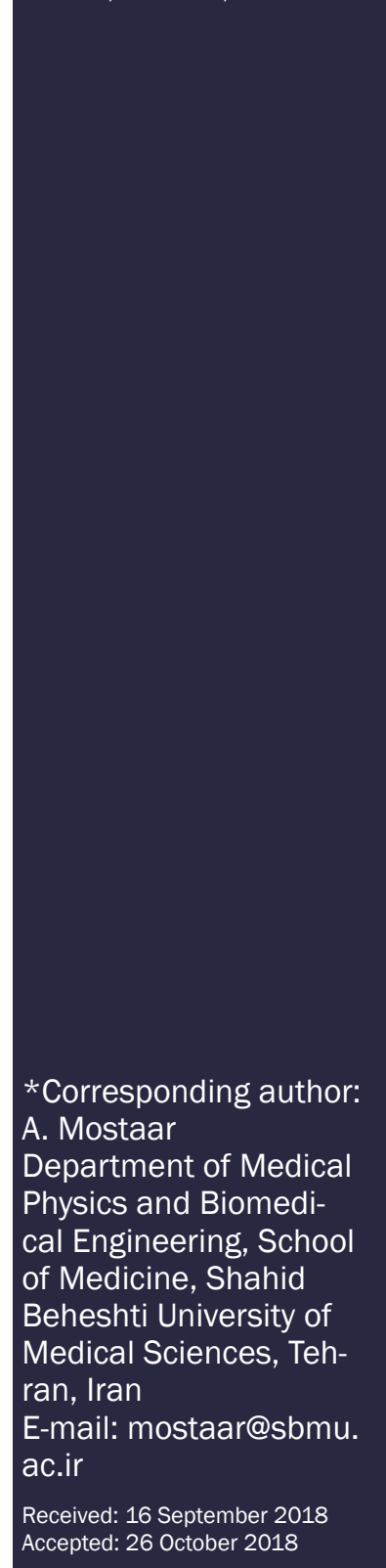


Yousefi Moteghaed N., Tabatabaeefar M., Mostaar A.

served while removing the noise. There are many works that use different methods for denoising medical images. There are two basic approaches to image denoising that include spatial filtering and transform domain filtering methods.

Most of the spatial filters are median based filtering techniques, which use the order statistical information of the pixels in the test window. There are so many other filters such as weighted median (WM) filter [1], Center Weighted Median (CWM) filter [2], Adaptive Center weighted median (ACWM) filter [3], Switching Median filter [4], Multi-State Median (MSM) filter [5], Directional Weighted Median filter [6], Second-Order Difference based Impulse Detection filter [7], Modified Directional Weighted Median based filter [8] and Modified Weighted based filter [9] which were proposed in the literature to remove impulses in the images. In the last decade, various denoising algorithms based on wavelet transform were introduced. Wavelets give a paramount performance in image denoising due to properties such as multi-resolution structure and sparsity [10]. Many studies which focused on the fusion of the denoising approaches have been done. These approaches were applied using both wavelet and curvelet transform [11], hybridization of wavelet and center weighted median filters [12] and also neural networks and deep learning [13] for eliminating the noise of the image.

Chawla et al. [14] proposed a method for removing the additive white Gaussian noise from the CT images in three phases; preprocessing, training and testing. The $\mathrm{CT}$ images were transformed using multi-wavelet transformation and then obtained multi-wavelet coefficients were the inputs of the adaptive Neuro-fuzzy inference system. In some other studies, the main objectives of denoising methods for obtaining the best estimate of the original image from its noisy version were based on using rearrangement of pixels and principal component analysis (PCA) [15], total varia- tion approach and complex dual-tree wavelet transform [16], signal-preserving technique based on spectral subtraction [17] and modified spatial filtration approach with adaptively decision on the masking center [18].

Ali et al. [19-21] proposed a noise reduction technique using thresholding and window based multi-wavelet transformation for CT images. This technique, especially was appropriate for removing additive white Gaussian noise and its results showed enhancement in the quality of CT images. Jia et al. [22] proposed a synchronization algorithm of contrast enhancement and denoising by applying a differential equation of limited adaptive histogram equalization and improvement PM model. Golshan et al. [23] presented an LMMSE-based method for the three-dimensional denoising of MR images which had been modeled MR data as random fields. So the method could construct a principled way for choosing the samples from a large portion of the given data. The parameters of the proposed filter are automatically selected from the estimated local SNR. In some studies, different hybrid filtering techniques such as a hybrid min filter and hybrid max filter for the removal of Gaussian noise, impulse noise or random noise from medical images were investigated. These methods were incorporated into spatial and frequency domain analysis. The quality of the noise reduction methods was determined by the statistical quantity measures [24-27]. Oulhaj et al [28] proposed a denoising task for different kinds of medical images by NLMeans algorithm and the effectiveness of the discussed algorithms were compared on the basis of SNR, PSNR, RMSE the mean structure similarity index. Devasena and Hemalatha [29] proposed a new scheme based on applying a series of filters by the combination of the Kernel, Sobel, and Low-pass (KSL) filtering techniques each of which was used to modify the stable estimate into greater agreement. In other studies, techniques that used genetic programming based approach and fuzzy ge- 
netic algorithm to find the optimal composite filters for removing all types of impulse noise from medical images were proposed [30-33]. In this study, we propose a sequence of filters for denosing of medical images that are selected by the hybrid genetic algorithm (GA) and particle swarm optimization algorithm (PSO). Then the quality of noise reduction is measured and compared between these algorithms.

\section{Material and Methods}

\section{a) Sequential filters}

In this analytical study, the sequence filters for producing models are selected by the hybrid optimization algorithm. Order filters such as median, max and min filter, Gaussian filter, average filter, unsharp filter, Wiener filter, $\log$ filter and sigma filter are 9 filters, which are used for the denoising images in this technique. We use a Wiener filter to produce better results than linear filtering. The Wiener filter (a type of linear filter) is applied to image adaptively.

In such a case that variance is small, Wiener filter performs more smoothly and is more selective than a comparable linear filter to preserve high-frequency parts and edge of an image. Also, when the noise is constant power additive noise, such as Gaussian noise, the Wiener filter works better. At least the model is implemented on medical images and the performances are evaluated by statistical quantitative measures. PSNR is most easily defined via the mean squared error (MSE). For a noise-free monochrome image $(\mathrm{I}(\mathrm{m} \times \mathrm{n}))$ and its noisy approximation $(\mathrm{N})$, the definition of MSE can be:

$$
M S E=\frac{1}{m n} \sum_{i=0}^{m-1} \sum_{j=0}^{n-1}[I(i, j)-N(i, j)]^{2}
$$

The PSNR (in $\mathrm{dB}$ ) is defined as:

$$
P S N R=10 \times \log _{10}\left(\frac{M A X_{I}^{2}}{M S E}\right)
$$

$M A X_{I}$ is the maximum possible pixel value of the image. Structural similarity (SSIM) index is a method for measuring the similarity between two images to improve on traditional methods such as PSNR and MSE. The SSIM metric is calculated on various windows of an image and is measured between two windows $\mathrm{x}$ and y of common size $K \times K$ :

$$
\operatorname{SSIM}(x . y)=\frac{\left(2 \mu_{x} \mu_{y}+c_{1}\right)\left(2 \sigma_{x y}+c_{2}\right)}{\left(\mu_{x}^{2}+\mu_{y}^{2}+c_{1}\right)\left(\sigma_{x}^{2}+\sigma_{y}^{2}+c_{2}\right)}
$$

Where $\mu_{x}, \mu_{y}$ is the average of $\mathrm{x}$ and $\mathrm{y}, \sigma_{x}^{2} \cdot \sigma_{y}^{2}$ is the variances of $\mathrm{x}$ and $\mathrm{y}$ and $\sigma_{x y}$ is the covariance of $\mathrm{x}$ and $\mathrm{y}$.

$$
\begin{aligned}
& c_{1}=\left(k_{1} L\right)^{2} \cdot c_{2}=\left(k_{2} L\right)^{2} \\
& L: \text { dynamic range of pixel value } \\
& k_{1}=0.01 \text { and } k_{2}=0.03
\end{aligned}
$$

\section{b) Proposed Algorithm}

In the proposed approach, genetic algorithm (GA) with the particle swarm optimization algorithm (PSO) is used as a denoising tool, to find the best sequence of filters for denoising task. Hybrid filter is a sequential filter where different filters are arranged in a sequence to obtain the best noise-free image. GA is a computational optimization method that searches all parts of the solution space with a different kind of solution or a group of features subset to find the best answer in each iteration. In GA, the searching process only needs to determine the value of the objective function at different points and also, no additional information like differentiation of function is needed. The most important operator in the GA algorithm is the crossover that creates a new population by a combination of chromosome depending on their selection. The parents that are selected for crossover, transfer their genes with each other to create new offspring. Crossover can eliminate fragmentation or genetic variation in the population. Mutation is another operator that creates the variety of solutions. In mutation operator, one gene may be removed from the subset or create a new gene that has been added to the population. 
PSO algorithm was developed by Eberhart and Kennedy in 1995 [34]. Each particle with a velocity adjusted by its own memory and its neighbors is moving in the search space to find the best solutions. The main difference is that there is no crossover and mutation operator in $\mathrm{PSO}$, hence it is more likely to be caught in a local minimum. But the best particle in PSO can remember and affect the other particles. So this property of the algorithm can lead to faster convergence. In contrast to the PSO algorithm, the chromosomes in the GA algorithm share the information with each other. Illustration of the pseudo code of algorithm for medical denoising application is shown in
Figure 1. One purpose is to increase the statistical quantity measures values by selecting the best sequence of filters using the proposed hybrid PSO/GA algorithm without the need of user trial-and-error for the parameter selection.

In the following we have to make the initial population. The initial parameters in genetic and PSO algorithm are shown in Table 1.

First, the populations with $\mathrm{N}$ chromosome are created randomly. The length of particles or chromosomes can be explained as, adding the number of features which have been selected based on filters and 6 additional genes which have been used for determination of

\begin{tabular}{|c|c|c|c|}
\hline $\begin{array}{l}\text { Pseudo code for GA } \\
\text { Initialization } \\
\text { for i=1:nPop } \\
\text { Position Evaluation } \\
\text { end } \\
\text { Sort Population } \\
\text { Store Best Solution } \\
\text { GA Main Loop } \\
\text { for it=1:MaxIt } \\
\text { Crossover } \\
\text { Select Parents } \\
\text { Apply Crossover } \\
\text { Evaluate Offspring } \\
\text { Mutation } \\
\text { Select Parent } \\
\text { Apply Mutation } \\
\text { Evaluate Mutant } \\
\text { Merge Population } \\
\text { Sort Population } \\
\text { Delete Extra Individuals } \\
\text { Update Best Solution } \\
\text { Store Best Cost } \\
\text { End }\end{array}$ & $\begin{array}{l}\text { Pseudo code for PSO } \\
\text { Parameters } \\
\text { determination } \\
\text { Initialization } \\
\text { for i=1:nPop } \\
\text { Initialize Position } \\
\text { Initialize velocity } \\
\text { Evaluation } \\
\text { end } \\
\text { Store Best Solution for } \\
\text { each particle } \\
\text { Store the global best } \\
\text { PSO Main Loop } \\
\text { for it=1:MaxIt } \\
\text { Update Velocity } \\
\text { Apply Velocity Bounds } \\
\text { Update Position } \\
\text { Evaluation } \\
\text { Update Personal Best } \\
\text { Update Global Best } \\
\text { end } \\
\text { Store Best Cost }\end{array}$ & $\begin{array}{l}\text { Pseudo code for } \\
\text { hybrid PSOGA } \\
\text { for it=1:MaxIt } \\
\text { PSO Operators } \\
\text { Update Velocity } \\
\text { Apply Velocity } \\
\text { Bounds } \\
\text { Update Position } \\
\text { Velocity Reflection } \\
\text { Evaluation } \\
\text { Update Personal Best } \\
\text { Update Global Best } \\
\text { GA Operators } \\
\text { Crossover } \\
\text { Select Parents } \\
\text { Evaluate Offsprings } \\
\text { Mutation } \\
\text { Merge Population } \\
\text { Sort Population } \\
\text { Update Worst Cost } \\
\text { Delete Extra } \\
\text { Individuals } \\
\text { Update Personal Best } \\
\text { Update Global Best } \\
\text { Store Best Cost } \\
\text { end }\end{array}$ & 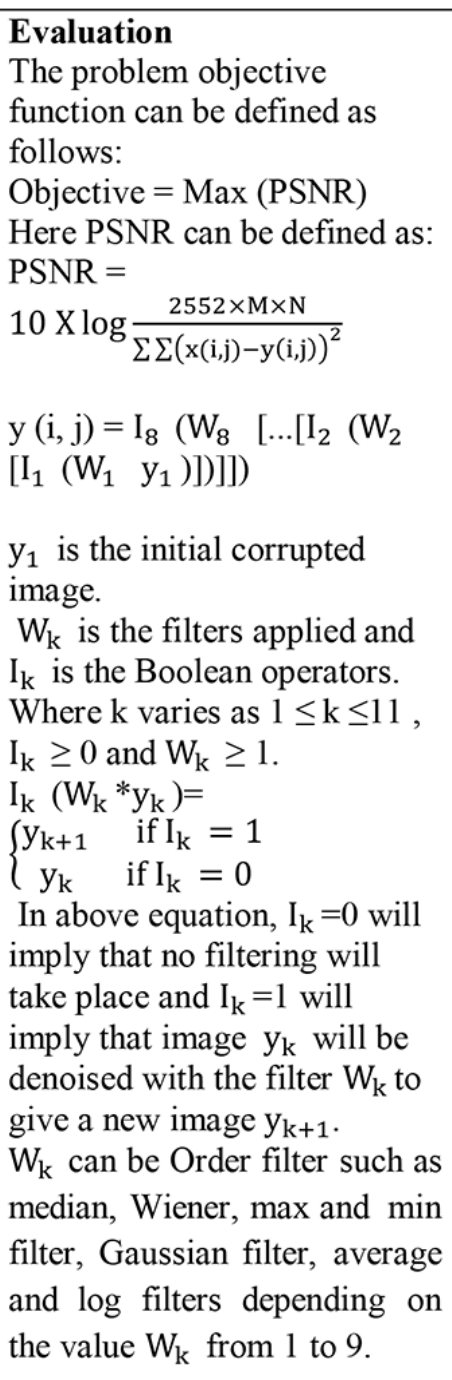 \\
\hline
\end{tabular}

Figure 1: pseudo-code of GA/PSO algorithm. 
Table 1: Initial parameters in GA/PSO.

\begin{tabular}{cc} 
PSO/GA parameters & Parameters \\
\hline Population & 10 \\
\hline Individual Length & 15 \\
\hline Number of features & 9 \\
\hline Number of iteration & 10 \\
\hline Inertia weight $(\mathrm{w})$ & 0.72 \\
\hline Acceleration constants & 1.49 \\
\hline Crossing rate & 0.9 \\
\hline Mutation rate & 0.1
\end{tabular}

optimum parameters of filters. Primary random and binary Initialization takes place first, in such a way that 1 shows the existence of a filter in the system and 0 is the meaning of not existing of that filter. Now each chromosome is a word of bits in two main parts. The first part is equal to the number of filters (segment 1 ), and the second part is used for determining and designing their parameters.

The second segment of a chromosome (two bits of the chromosome) determines the size value for 9 types of filters such as averaging, min, max, median, Gaussian and Wiener. The third segment shows the parameters of the order filter. We assigned 2 and 4 bits for the second and the third parts respectively, which were converted to the decimal number during the procedure.

The fitness values for all particles have to be calculated in order to determine the functionality of each particle, which is called validation of particles. In this step, the fitness values for all particles are calculated to determine the functionality of each particle, which is called validation of particles.

For PSO approach we propose the updating procedure of the best particle as $x^{\text {gbest }}$ and the best personal memories of each particle $x^{\text {ibest }}$ in the whole population. Also, velocity and position of the particles are updated based on the equation below:

$v_{j}^{i}[t+1]=w v_{j}^{i}[t]+C_{1} r_{1}\left(x_{j}^{i \text { ibest }}[t]-x_{j}^{i}[t]\right)+C_{2} r_{2}\left(x_{j}^{\text {gbest }}[t]-x_{j}^{i}[t]\right)(4)$
In this paper, we have used a binary PSO algorithm [35], so the velocity of the updating procedure would be different. In the binary algorithm, the concept of the velocity is changing to means of probability and the velocity is explaining the probability of the existence of 1 in position. The amount of the velocity is considered between 0 and 1 which explains the probability of being 1 in a position. So the velocity would be calculated from the equation (4) in the real state. Then the velocity has been mapped to the value of 0 and 1 by limiting sigmoid function and at the final state the position of the particle (i) is determined by the equation below:

$s(z)=\frac{1}{1+e^{-v_{j}^{i}}} \quad x_{j}^{i}[t]=\left\{\begin{array}{cc}1 & . \sigma<s\left(v_{j}^{i}[t]\right) \\ 0 & \text { otherwise }\end{array}\right.$

It is to consider that, for increasing the velocity of the system divergence, the limitation issue of velocity in the system can be considered based on maximum and minimum velocity. It is important to note that in genetic operators, there is no discussion on speed changes or the best memory of offspring. Hence we have determined the best memory of offspring based on the best memory of parents, which have the best fitness value. The speed can be selected randomly.

The roulette-wheel approach has been used for selection in the proposed method. After the steps for parent selection, the steps executing the genetic operators commence. Single point, double point and uniform crossover by random probability are used to benefit these crossover methods simultaneously. Figure 2 is the flowchart of the whole process. This flowchart summarizes how the system works and the relationships between the optimization part and the evaluation part are shown.

The proposed method was tested on CT and MRI images. We applied the composite of different types of noise such as salt and pepper noise, speckle noise and Gaussian noise to each image to make it noisy in two models. The first model is the combination of about $5 \%$ of each 


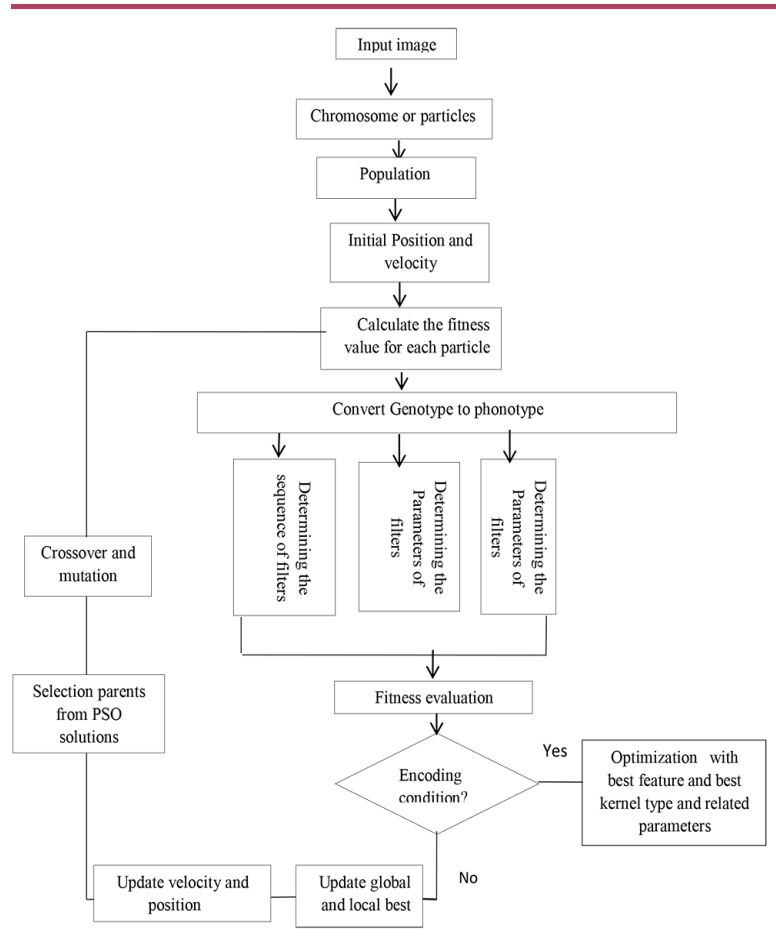

Figure 2: Medical image denoising algorithm using hybrid Genetic Algorithm (GA) and particle swarm optimization applied to sequential filters.

noise (Gaussian noise with 0.01 variance, salt, and pepper noise with a noise density of 0.05 and speckle noise with 0.04 variance). The second model is combination of about $10 \%$ of each noise (Gaussian noise with 0.1 variance, salt, and pepper noise with noise density of 0.1 and speckle noise with variance of 0.1 ). Salt and pepper noise is a form of noise in which represents itself as randomly occurring white and black pixels. A spike noise achieves the intensity values of random pixels with either maximum or minimum values. Gaussian noise has a probability density function (PDF) of the normal distribution.

\section{Results}

Here we used PSNR, RMSE and SSIM values as our fitness function of the PSO and GA algorithms which is directly proportional to these values. PSNR is a popular quality metric because it is easy and fast to calculate. In gen- eral, a higher quality image should correlate to a higher PSNR value. The results of the algorithm on datasets are shown in Figure 3 for noise model 1 and Figure 4 for noise model 2.

\section{Discussion}

To investigate the accuracy of the proposed PSO/GA hybrid algorithm, the results were examined in greater detail. For each MRI and CT datasets, the evaluation parameters such as PSNR, RMSE, and SSIM are calculated. Also, the comparison between these two optimization algorithms to find the best parameters and the best sequence of the filter to get the best free noise images are demonstrated in Table 2 for noise model 1 and Table 3 for noise model 2.

Another comparison was made between the proposed algorithm and other presented algorithms. Table 4 shows the results of the comparison based on PSNR and RMSE value.

\section{Conclusion}

Performance of proposed denoising algorithms is measured using quantitative and statistical measures as well as visual quality of the images. There have been several published methods, each of which approach has its assumptions, advantages, and limitations. An appropriate and ideal denoising procedure requires a priori knowledge of the noise, whereas a practical procedure may not have the sufficient information about the noise model or the variance of the noise. In this paper, we present a new approach by applying hybrid of GA and PSO algorithms as a denoising process of finding the best sequence of filters. So it seems better results for image denoising are achievable using a series of filters that are organized by the proposed algorithm. The results of the present study provide a comprehensive comparison of the proposed algorithm and those from previously published sources.

\section{Acknowledgment}

This article was derived from the thesis of 

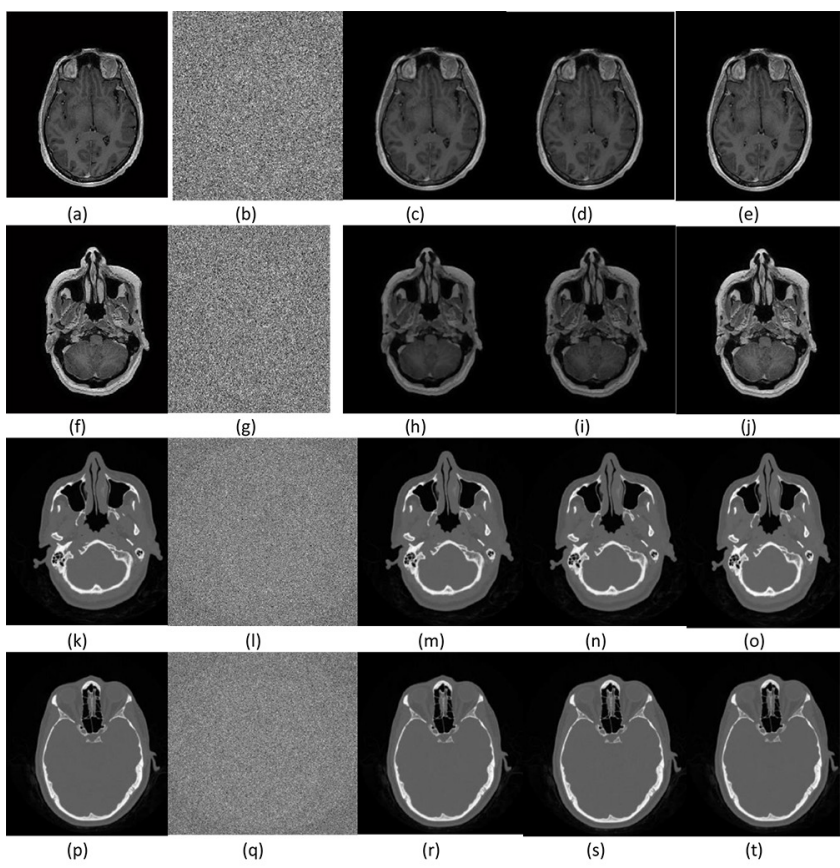

Figure 3: Outcome of denoising methods on the MRI and CT images of head with noise model 1: $(a, f, k, p)$ are original images, $(b, g, l, q)$ are noisy images, $(c, h, m, r)$ are denoised images by GA method, $(d, i, n, s)$ are denoised images by PSO method and $(e, j, o, t)$ are denoised images by hybrid PSOGA method.
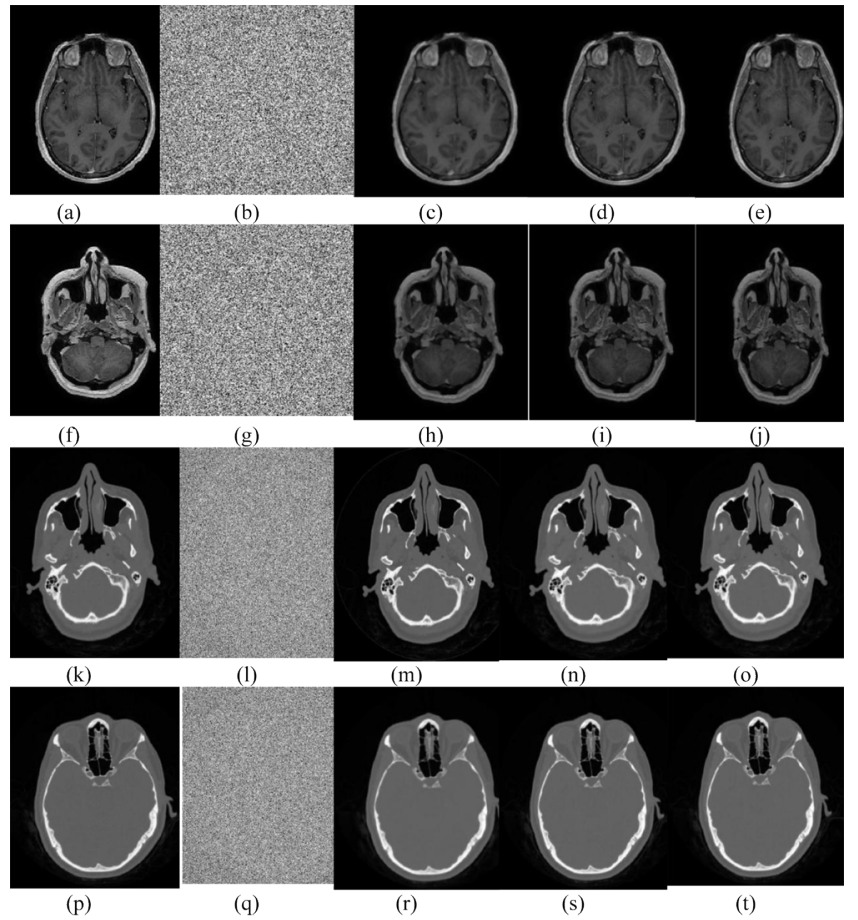

Figure 4: Outcome of denoising methods on sample MR and CT images of head with noise model 2: $(a, f, k, p)$ are original images, $(b, g, l, q)$ are noisy images, $(c, h, m, r)$ are denoised images by GA method, $(d, i, n, s)$ are denoised images by PSO method and $((e, j, o, t))$ are denoised images by hybrid PSOGA method. 
Yousefi Moteghaed N., Tabatabaeefar M., Mostaar A.

Table 2: Comparison of results between proposed methods as PSNR, SSIM and RMSE values in $\mathrm{dB}$ for noise model 1 .

\begin{tabular}{ccccc} 
& Datasets & PSNR & RMSE & SSIM \\
\hline \multirow{3}{*}{ Noisy image } & MRI & $14.85 \pm 0.016$ & $180.286 \pm 0.0150$ & $0.0314 \pm 0.00000816$ \\
\cline { 2 - 5 } & MRI & $14.87 \pm 0.0235$ & $180.27 \pm 0.0377$ & $0.0311 \pm 0.000298$ \\
\cline { 2 - 5 } & CT & $14.63 \pm 0.0122$ & $180.25 \pm 0.0215$ & $0.0311 \pm 0.000189$ \\
\cline { 2 - 5 } GA & CT & $14.83 \pm 0.0192$ & $180.26 \pm 0.0153$ & $0.0312 \pm 0.000182$ \\
\hline \multirow{3}{*}{ PSO } & MRI & $62.28 \pm 1.76$ & $41.59 \pm 8.71$ & $0.9969 \pm 0.00036$ \\
\cline { 2 - 5 } & MRI & $57.01 \pm 0.825$ & $54.85 \pm 2.39$ & $0.9982 \pm 0.0012$ \\
\hline & CT & $56.85 \pm 3.64$ & $35.41 \pm 1.03$ & $0.9992 \pm 0.000692$ \\
\hline & CT & $60.38 \pm 3.48$ & $32.73 \pm 2.42$ & $0.9994 \pm 0.000351$ \\
\hline & MRI & $58.96 \pm 3.80$ & $37.79 \pm 9.35$ & $0.9985 \pm 0.0013$ \\
\hline \multirow{3}{*}{ GAPSO } & MRI & $63.72 \pm 3.82$ & $36.80 \pm 0.0550$ & $0.9991 \pm 0.0013$ \\
\hline & CT & $60.89 \pm 3.52$ & $18.55 \pm 5.41$ & $0.9994 \pm 0.000541$ \\
\hline & CT & $66.194 \pm 1.97$ & $12.73 \pm 0.49$ & $0.9996 \pm 0.0002$ \\
\hline & MRI & $63.07 \pm 0.0134$ & $38.47 \pm 4.49$ & $0.9981 \pm 0.0010$ \\
\hline & MRI & $63.5 \pm 2.82$ & $36.80 \pm 0.0550$ & $0.9997 \pm 0.000577$ \\
\cline { 2 - 5 } & CT & $63.28 \pm 1.69$ & $13.89 \pm 0$ & $0.9998 \pm 0.000294$ \\
\cline { 2 - 5 } & CT & $65.222 \pm 0.39$ & $12.31 \pm 0$ & $0.9997 \pm 0.00066$
\end{tabular}

Table 3: Comparison of results between proposed methods as PSNR, SSIM and RMSE values in $\mathrm{dB}$ for noise model 2.

\begin{tabular}{ccccc} 
& Datasets & PSNR & RMSE & SSIM \\
\hline \multirow{3}{*}{ Noisy image } & MRI & $11.62 \pm 0.0150$ & $180.536 \pm 0.0089$ & $0.0156 \pm 0.00025$ \\
\cline { 2 - 5 } & MRI & $11.6 \pm 0.0071$ & $180.546 \pm 0.0167$ & $0.0156 \pm 0.0000816$ \\
\cline { 2 - 5 } & CT & $11.63 \pm 0.0122$ & $180.53 \pm 0.0192$ & $0.0157 \pm 0.0000957$ \\
\cline { 2 - 5 } GA & CT & $11.61 \pm 0.0164$ & $180.54 \pm 0.0089$ & $0.0158 \pm 0.00001$ \\
\hline \multirow{3}{*}{ PSO } & MRI & $54.67 \pm 1.3$ & $66.85 \pm 2.5$ & $0.9955 \pm 0.0013$ \\
\hline & MRI & $56.73 \pm 1.26$ & $63.16 \pm 6.28$ & $0.9990 \pm 0.001$ \\
\hline & CT & $56.04 \pm 3.26$ & $40.33 \pm 4.85$ & $0.9967 \pm 0.00096$ \\
\hline & CT & $58.86 \pm 3.23$ & $38.76 \pm 1.83$ & $0.9989 \pm 0.0000577$ \\
\hline & MRI & $61.47 \pm 3.58$ & $65.47 \pm 0.73$ & $0.9965 \pm 0.00036$ \\
\hline \multirow{2}{*}{ GAPSO } & $60.75 \pm 3.69$ & $61.12 \pm 3.34$ & $0.9990 \pm 0.000635$ \\
\hline & CT & $62.06 \pm 1.76$ & $25.91 \pm 5.76$ & $0.9985 \pm 0.00015$ \\
\hline & CT & $63.78 \pm 2.066$ & $20.40 \pm 5.90$ & $0.9995 \pm 0.000404$ \\
\hline & MRI & $59.63 \pm 4.002$ & $44.37 \pm 9.83$ & $0.9977 \pm 0.00115$ \\
\hline & MRI & $63.50 \pm 2.81$ & $47.64 \pm 9.94$ & $0.9992 \pm 0.000152$ \\
\hline & CT & $63.48 \pm 1.88$ & $16.43 \pm 4.91$ & $0.9992 \pm 0.0011$ \\
\hline & CT & $64.08 \pm 1.63$ & $20.40 \pm 5.90$ & $0.9993 \pm 0.00023$
\end{tabular}


Table 4: Comparison of results between proposed hybrid method and other works as PSNR and RMSE values in $\mathrm{dB}$.

\begin{tabular}{lcclc}
\multirow{2}{*}{ Proposed works } & \multicolumn{2}{c}{ PSNR } & \multicolumn{2}{c}{ RMSE } \\
\cline { 2 - 5 } & MRI & CT & MRI & CT \\
\hline Kota NS, et al [11] & $14-71$ & - & - & - \\
\hline Umamaheswari J, et al [12] & $31-38$ & - & - & - \\
\hline Chawla P, et al [14] & $14-18$ & - & - & - \\
\hline Mredhula L, et al [15] & - & $23-25$ & - & - \\
\hline Lalitha YS, et al [18] & $17-28$ & & - & - \\
\hline Ali SA, et al [19] & - & $36-39$ & - & - \\
\hline Syed AA [20] & - & $23-29$ & - & - \\
\hline Ilango G, et al [24] & - & $36.5-61.2$ & - & $0.2-3.8$ \\
\hline Marudhachalam R, et al [25] & $25-59$ & - & $0.5-13$ & - \\
\hline Bharathi D, et al [26] & $27-45$ & - & $1.6-3.2$ & - \\
\hline Marudhachalam R, et al [27] & $25-45$ & - & $1-13$ & - \\
\hline Sharif M, et al [30] & 22.54 & - & 20.68 & - \\
\hline Anisha KK, et al [31] & $22-26$ & - & - & - \\
\hline Our proposed algorithm & $59-63$ & $63-65$ & $36-47$ & $12-20$
\end{tabular}

Niloofar Yousefi Moteghaed in the Biomedical and Medical Physics Department at the Shahid Beheshti University of Medical Sciences. (Registration No:140M).

\section{Conflict of Interest}

None

\section{References}

1. Yli-Harja 0, Astola J, Neuvo Y. Analysis of the properties of median and weighted median filters using threshold logic and stack filter representation. IEEE Transactions on Signal Processing. 1991;39:395-410. doi: 10.1109/78.80823.

2. Ko S-J, Lee YH. Center weighted median filters and their applications to image enhancement. IEEE transactions on circuits and systems. 1991;38:98493. doi: $10.1109 / 31.83870$.

3. Chen T, Wu HR. Adaptive impulse detection using center-weighted median filters. IEEE Signal Processing Letters. 2001;8:1-3. doi: 10.1109/97.889633.

4. Chen T, Ma KK, Chen LH. Tri-state median filter for image denoising. IEEE Trans Image Process. 1999;8:1834-8. doi: 10.1109/83.806630. PubMed PMID: 18267461.

5. Chen T, Wu HR. Space variant median filters for the restoration of impulse noise corrupted images. IEEE transactions on circuits and systems II: analog and digital signal processing. 2001;48:784-9. doi: $10.1109 / 82.959870$.

6. Dong Y, Xu S. A new directional weighted median filter for removal of random-valued impulse noise. IEEE signal processing letters. 2007;14:193-6. doi: 10.1109/lsp.2006.884014.

7. Sa PK, Dash R, Majhi B, editors. Second order difference based detection and directional weighted median filter for removal of random valued impulsive noise. 2009 International Conference on Industrial and Information Systems (ICIIS). Sri Lanka: IEEE; 2009. p. 362-364. doi: 10.1109/iciinfs.2009.5429836.

8. Mandal J, Sarkar A, editors. A Novel Modified Directional Weighted Median Based Filter for Removal of Random Impulse Noise (MDWMF). 2010 International Symposium on Electronic System Design. Bhubaneswar, India: IEEE; 2010. p. 230-234. doi: 10.1109/ised.2010.51.

9. Mandal J, Sarkar A, editors. A Modified Weighted Based Filter for Removal of Random Impulse Noise (MWB). 2011 Second International Conference on Emerging Applications of Information Technology. Kolkata, India: IEEE; 2011. P. 173-176. doi: 10.1109/eait.2011.77.

10. Agarwal S, Singh 0, Nagaria D. Analysis and Comparison of Wavelet Transforms for Denoising MRI Image. Biomedical and Pharmacology Journal. 2017;10:831-6. doi: 10.13005/bpj/1174.

11. Kota NS, Reddy GU. Fusion based Gaussian noise removal in the images using curvelets and wavelets with Gaussian filter. Int J Image Process 
Yousefi Moteghaed N., Tabatabaeefar M., Mostaar A.

\section{(IJIP). 2011;5:456-68.}

12. Umamaheswari J, Radhamani DG. Hybrid Denoising method for removal of mixed noise in medical images. International Journal of Advanced Computer Science and Applications. 2012;3. doi: 10.14569/ijacsa.2012.030507.

13. Jifara W, Jiang F, Rho S, Cheng M, Liu S. Medical image denoising using convolutional neural network: a residual learning approach. The Journal of Supercomputing. 2017:1-15. doi: 10.1007/ s11227-017-2080-0.

14. Chawla P, Mittal R, Grewal K. Hybrid filtering technique for image denoising using artificial neural network. International Journal of Engineering and Advanced Technology (IJEAT). 2012;1:36-40.

15. Mredhula L, Dorairangaswamy MA. A combined pca model for denoising of ct images. Elysium Journal. 2014;1:46-50.

16. Raj VP, Venkateswarlu T. Denoising of medical images using image fusion techniques. Signal \& Image Processing. 2012;3:65. doi: 10.5121/ sipij.2012.3406.

17. Priyadharsini B. A novel noise filtering technique for denoising MRI images. ICGICT. 2014;2:242833.

18. Lalitha Y, Latte MV. A novel approach noise filtration for MRI image sample in medical image processing. International Journal of Computer Science and Communication. 2011;2:359-63.

19. Ali SA, Vathsal S. A GA based Window Selection Methodology to Enhance Window based Multi wavelet transformation and thresholding aided CT image denoising technique. alnternational Journal of Computer science and information security. 2010;7(2): 280-288.

20. Syed AA. CT Image Denoising Technique using GA aided Window-based Multiwavelet Transformation and Thresholding with the Incorporation of an Effective Quality Enhancement Method. International Journal of digital contenttechnology and its application. 2010;4(4):75-88. doi: 10.4156/jdcta.vol4. issue4.8.

21. Ali SA, Vathsal S, Kishore KL. An efficient denoising technique for CT images using windowbased multi-wavelet transformation and thresholding. European Journal of Scientific Research. 2010;48:315.

22. Di Jia FH, Yang J, Zhang Y, Zhao D, Yu G. A synchronization algorithm of MRI denoising and contrast enhancement based on PM-CLAHE model. International Journal of Digital Content Technology and its Applications. 2010;4(6):144-17. doi: 10.4156/jdcta.vol4.issue6.17.

23. Golshan HM, Hasanzadeh RP, Yousefzadeh SC. An MRI denoising method using image data redundancy and local SNR estimation. Magn Reson Imaging. 2013;31:1206-17. doi: 10.1016/j. mri.2013.04.004. PubMed PMID: 23668996.

24. Ilango G, Marudhachalam R. New hybrid filtering techniques for removal of Gaussian noise from medical images. ARPN Journal of Engineering and Applied Sciences. 2011;6:8-12.

25. Marudhachalam R, Ilango G. A New Hybrid Filtering Technique for Removal of Impulse Noise From Digital Images. International Journal of Mathematical Archive EISSN 2229-5046. 2012;3.

26. Bharathi D, Govindan SM. A New Hybrid Approach for Denoising Medical Images. Advances in Computing and Information Technology: Springer; 2013. p. 905-14. doi: 10.1007/978-3-642-315527_92.

27. Marudhachalam R, Ilango G. Fuzzy hybrid filtering techniques for removal of random noise from medical images. International Journal of Computer Applications. 2012;38:15-8. doi: 10.5120/45716732.

28. Oulhaj H, Amine A, Rziza M, Aboutajdine D, editors. Noise Reduction in Medical Images-comparison of noise removal algorithms. 2012 International Conference on Multimedia Computing and Systems. Tangier, Morocco: IEEE; 2012. p. 344-349. doi: 10.1109/ICMCS.2012.6320218.

29. Devasena CL, Hemalatha M. Noise removal in magnetic resonance images using hybrid KSL filtering technique. Methodology. 2011;27. doi: 10.5120/3324-4571.

30. Sharif M, Jaffar MA, Mahmood MT. Optimal composite morphological supervised filter for image denoising using genetic programming: Application to magnetic resonance images. Engineering $A p$ plications of Artificial Intelligence. 2014;31:78-89. doi: 10.1016/j.engappai.2013.11.011.

31. Anisha K, Wilscy M. Impulse noise removal from medical images using fuzzy genetic algorithm. The International Journal of Multimedia \& Its Applications. 2011;3:93. doi: 10.5121/ijma.2011.3408.

32. Taher GM, El Taweal G, Wahed ME, Fouad A. Image fusion approach with noise reduction using $\mathrm{Ge}-$ netic Algorithm. International Journal of Advanced Computer Science and Applications. 2013;4(11).

33. De Paiva JL, Toledo CF, Pedrini H. An approach based on hybrid genetic algorithm applied to image denoising problem. Applied Soft Computing. 2016;46:778-91. doi: 10.1016/j.asoc.2015.09.013.

34. Eberhart R, Kennedy J, editors. A new optimizer using particle swarm theory. MHS'95. Proceedings of the Sixth International Symposium on Micro Machine and Human Science. Nagoya, Japan: IEEE; 1995. p. 39-43. doi: 10.1109/MHS.1995.494215.

35. Kennedy J, Eberhart RC. A discrete binary version of the particle swarm algorithm. 1997 IEEE International Conference on Systems, Man, and Cybernetics. Computational Cybernetics and Simulation. Orlando, FL, USA: IEEE; 1997;5:4104-8. doi: 10.1109/icsmc.1997.637339. 